\title{
Circulating endothelial cells are increased in chronic myeloid leukemia blast crisis
}

\author{
C.R.T. Godoy ${ }^{1}$, D. Levy ${ }^{2}$, V. Giampaoli ${ }^{3}$, D.A.F. Chamone ${ }^{1}$, S.P. Bydlowski ${ }^{2}$ and J. Pereira ${ }^{1}$ \\ ${ }^{1}$ Faculdade de Medicina, Universidade de São Paulo, São Paulo, SP, Brasil \\ ${ }^{2}$ Laboratório de Genética e Hematologia Molecular, Universidade de São Paulo, São Paulo, SP, Brasil \\ ${ }^{3}$ Departamento de Estatística, Instituto de Matemática e Estatística, Universidade de São Paulo, São Paulo, SP, Brasil
}

\begin{abstract}
We measured circulating endothelial precursor cells (EPCs), activated circulating endothelial cells (aCECs), and mature circulating endothelial cells (mCECs) using four-color multiparametric flow cytometry in the peripheral blood of 84 chronic myeloid leukemia (CML) patients and 65 healthy controls; and vascular endothelial growth factor (VEGF) by quantitative realtime PCR in $50 \mathrm{CML}$ patients and 32 healthy controls. Because of an increase in mCECs, the median percentage of CECs in CML blast crisis $(0.0146 \%)$ was significantly higher than in healthy subjects $(0.0059 \%, \mathrm{P}<0.01)$ and in the accelerated phase $(0.0059 \%, P=0.01)$. There were no significant differences in the percentages of CECs in chronic- or active-phase patients and healthy subjects $(\mathrm{P}>0.05)$. In addition, VEGF gene expression was significantly higher in all phases of CML: 0.245 in blast crisis, 0.320 in the active phase, and 0.330 in chronic phase patients than it was in healthy subjects $(0.145)$. In conclusion, CML in blast crisis had increased levels of CECs and VEGF gene expression, which may serve as markers of disease progression and may become targets for the management of CML.
\end{abstract}

Key words: Circulating endothelial cells; Vascular endothelial growth factor; Chronic myeloid leukemia; Flow cytometry; Angiogenesis

\section{Introduction}

Angiogenesis is essential for the development and progression of solid malignancies because it supports the nutrition and oxygenation of malignant cells $(1,2)$. It is an active, multistep process that includes the activation, proliferation, and migration of endothelial cells (ECs), and the activation of cytokines (3). Vascular endothelial growth factor (VEGF) is particularly important in this process through its interactions with tyrosine kinase receptors (TKRs), VEGF receptor (VEGFR)-1, and VEGFR2. The latter is an important regulator of $E C$ recruitment, proliferation and survival $(1,2,4)$. The level of circulating endothelial cells (CECs) in peripheral blood has been proposed as a noninvasive marker of angiogenesis (5).

An increase in the number of CECs has been reported in multiple myeloma (6) and myelodysplasia (4), but the prognostic value and meaning of this remain unknown. Rigolin et al. (7) found that CECs and endothelial precursor cells (EPCs) were increased in chronic lymphocytic leukemia compared to healthy controls, and that high levels of CECs were correlated with more aggressive disease and shorter time to first treatment. In addition, Ibrahim et al. (8) reported higher levels of CECs in the peripheral blood of acute myeloid leukemia patients than in healthy subjects. CEC levels were related to response to therapy and disease progression (8).

Chronic myeloid leukemia $(\mathrm{CML})$ is a malignant clonal disorder of hematopoietic stem cells (9) that has been associated with high microvessel density and high serum VEGF levels. Indeed, the positive bcr-abl leukemic cells characteristic of CML may stimulate the migration of ECs from the bone marrow to the peripheral blood, mainly in blast crisis (BC). CEC levels in different phases of $\mathrm{CML}$ have not been extensively studied (10-13).

The aim of this study was to quantify CEC levels and VEGF gene expression in different phases of $\mathrm{CML}$ compared to healthy subjects. Our hypothesis was that CEC levels and VEGF gene expression were related to CML progression.

\section{Subjects and Methods}

\section{Patients}

Following approval by the Ethics Board of the Hospital das Clínicas, Faculdade de Medicina, Universidade de

Correspondence: J. Pereira: <julianapereira29@hotmail.com>. 
Sao Paulo, 84 patients with CML were enrolled in the study after providing written informed consent. Thirty-one $(36.9 \%)$, with a median age of 46 years, were in the chronic phase (CP); $23(27.4 \%)$ with a median age of 52 years were in blast crisis (BC); and 30 (35.7\%) with a median age of 58 years were in the active phase (AP). VEGF gene expression was analyzed in 65 individuals, 25 $(26 \%)$ in CP (median age 51 years); 14 (14\%) in BC (median age 53 years); and $26(27.0 \%)$ in AP (median age 58 years). All patients were classified according to the World Health Organization classification of myeloproliferative disorders after evaluation of peripheral blood, bone marrow aspirate, bone marrow biopsy, karyotyping and molecular analysis.

A control group of 50 volunteer apheresis platelet donors, 25 men and 25 women with a median age of 44 years (range 19 to 79 years) was evaluated for CEC. VEGF gene expression was determined in 32 volunteer platelet donors ( 17 men and 15 women with a median age of 49 years; range 21 to 77 years). No female controls were evaluated during a menstrual period. Patients diagnosed with $\mathrm{BC}$ were tested by flow cytometric immunophenotyping to determine whether the crisis was acute lymphoid or myeloid in lineage. Analysis of CECs and VEGF gene expression was carried out in different numbers of samples because performing the required molecular techniques was not feasible in some samples.

\section{Blood samples}

Venous blood samples $(10 \mathrm{~mL})$ from $\mathrm{CML}$ patients and control subjects were collected in pyrogen-free tubes containing ethylenediaminetetraacetic acid (EDTA).

\section{Characterization of CECs}

Procedure for flow cytometry. Eighty-four CML patients and 50 healthy volunteers were tested. The different subpopulations of CECs were assayed by fourcolor multiparametric flow cytometry using a specific monoclonal antibody (MoAb) panel and a lyze/wash technique previously described by Mancuso et al. (14).

In brief, $1 \times 10^{6}$ cells were added to each of three different tubes. One contained $10 \mu \mathrm{L}$ of a phycoerythrincyanine 5.1-conjugated anti-CD45 clone J33 antibody (CD45/PC5, 1:10; Immunotech, France). The second tube contained $10 \mu \mathrm{L}$ fluorescein isothiocyanate (FITC)conjugated anti-CD146 clone OJ79C antibody (CD146/ FITC; Serotec, UK), $10 \mu \mathrm{L}$ of phycoerythrin-conjugated anti-CD34 class III clone BIRMA-K3 antibody (CD34/PE; DakoCytomation, USA), $10 \mu \mathrm{L}$ CD45/PC5 (1:10), and $10 \mu \mathrm{L}$ allophycocyanin-conjugated anti-CD133 clone 293C3 antibody (CD133/APC; Milteny Biotec, USA). The third tube contained $10 \mu \mathrm{L}$ of CD146/FITC, $20 \mu \mathrm{L}$ of PEconjugated anti-CD62e clone TEA2/1 antibody (CD62e/ PE; BD Bioscience, USA), $10 \mu \mathrm{L} \mathrm{CD45/PC5} \mathrm{(1:10),} \mathrm{and}$ $10 \mu \mathrm{L}$ CD133/APC. All tubes were incubated in the dark for $20 \mathrm{~min}$ followed by red cell lysis by the addition of
$2000 \mu \mathrm{L}$ of $\mathrm{BD}$ lyzing solution diluted $1: 10$ in deionized water. The tubes were then centrifuged at $1000 \mathrm{~g}$ for $3 \mathrm{~min}$ and washed twice with $2000 \mu \mathrm{L}$ of phosphatebuffered saline (PBS) containing $0.1 \%$ azide. Cells were resuspended in $400 \mu \mathrm{L} 1 \%$ formaldehyde and 100,000 events per tube were read on a FACSCalibur flow cytometer (BD Biosciences, USA) and analyzed using CellQuest Pro software (BD Biosciences). Flow cytometer performance was monitored daily using CaliBRITE microbeads and reagent with the FACSComp software (BD Biosciences). All procedures were performed in duplicate to verify the reproducibility of the results.

Validation of MoAb. All MoAb tests were validated with human umbilical vein endothelial cells (HUV-EC-C, ATCC CRL-1730; Manassas, USA) cultivated at our laboratory in 199/EBS medium complemented with $10 \%$ fetal bovine serum, $100 \mathrm{U} / \mathrm{mL}$ penicillin, and $100 \mu \mathrm{g} / \mathrm{mL}$ streptomycin.

Analysis of endothelial cells. We first selected all living cells in a side-scatter (SSC)/CD45 plot to exclude platelets, dead cells, and debris. Then, we gated around a region corresponding to CD45 negative and low SSC in order to exclude hematopoietic cells (Figure 1) (14-16). Subsequently, we looked for CD146, CD34, CD62e, and CD133 expression in other 2D fluorescence plots from R2. EPCs were considered as $\mathrm{CD} 45^{-/ \mathrm{dim}} / \mathrm{CD} 146^{+} /$ CD $133^{+} / \mathrm{CD}_{34}{ }^{+} / \mathrm{CD} 62 \mathrm{e}^{-/+}$and mature (m)CECs as $\mathrm{CD}^{-/ \mathrm{dim}} / \mathrm{CD} 146^{+} / \mathrm{CD} \mathrm{e}^{+/-} / \mathrm{CD} 133^{-} / \mathrm{CD}^{-} 4^{-}$(Figure 2). The presence of the CD62e antigen characterized activated EPCs and mCECs. CECs were reported as a percentage of the total events after the exclusion of debris. To prevent bias, only percentage values were used in statistical analyses because the leukocyte count was highly variable in each group. The medians were $6.2 \times 10^{9} / \mathrm{L}$ in the control group, $21.2 \times 10^{9} / \mathrm{L}$ in the CPCML group, $87.5 \times 10^{9} / \mathrm{L}$ in the $\mathrm{BC}-\mathrm{CML}$ group, and $6.1 \times 10^{9} / \mathrm{L}$ in the AP-CML group.

\section{Detection of VEGF by real-time quantitative PCR}

Mononuclear isolates. Mononuclear cells were isolated from the buffy coats of venous blood samples obtained from $65 \mathrm{CML}$ patients and 32 healthy donors. The steps included dilution of whole blood with an equal volume of PBS. Four parts of diluted blood was added carefully to three parts Ficoll-Paque Plus (GE Healthcare Life Science, UK) and centrifuged at $400 \mathrm{~g}$ for $30 \mathrm{~min}$. The buffy coat cells were isolated by washing twice in PBS with centrifugation for $10 \mathrm{~min}$ at $100 \mathrm{~g}$. Then, monocytes were counted in a Neubauer chamber, and $1 \times 10^{7}$ cells were frozen at $-20^{\circ} \mathrm{C}$ in $1.0 \mathrm{~mL}$ of Trizol reagent (Invitrogen, USA).

RNA isolation. Cells were thawed at room temperature, with the subsequent addition of $200 \mu \mathrm{L}$ of chloroform followed immediately by homogenization, and then resting for $3 \mathrm{~min}$ at room temperature. The homogenate was centrifuged at $11,800 \mathrm{~g}$ for $15 \mathrm{~min}$ at 

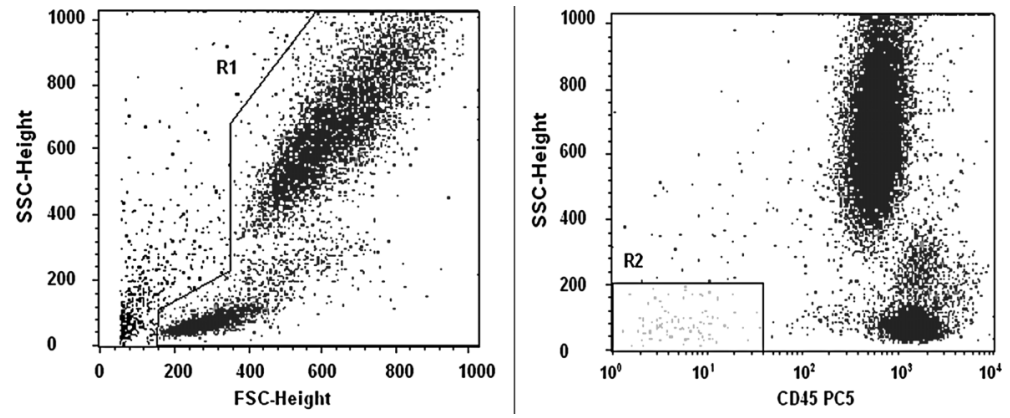

Figure 1. Left, Forward side scatter (FSC) vs side scatter (SSC) cytogram in dot plots showing region 1 (R1) gating mononuclear cells and excluding debris and (right) $\mathrm{CD} 45^{-/ \mathrm{dim}}$ vs SSC. R2: region 2; PC5: phycoerythrin-cyanin 5.1. $4^{\circ} \mathrm{C}$. The supernatant was transferred to a new $1.5 \mathrm{~mL}$ previously identified tube and $500 \mu \mathrm{L}$ of isopropanol was gently added. Following incubation for $10 \mathrm{~min}$ at room temperature to promote precipitation of RNA, the mixture was centrifuged at $11,800 \mathrm{~g}$ for $10 \mathrm{~min}$ at $4^{\circ} \mathrm{C}$. The supernatant was discarded and the RNA was washed in $1 \mathrm{~mL}$ of $75 \%$ ethanol diluted in diethylpyrocarbonate (DEPC)-treated water. After centrifugation for $5 \mathrm{~min}$ at
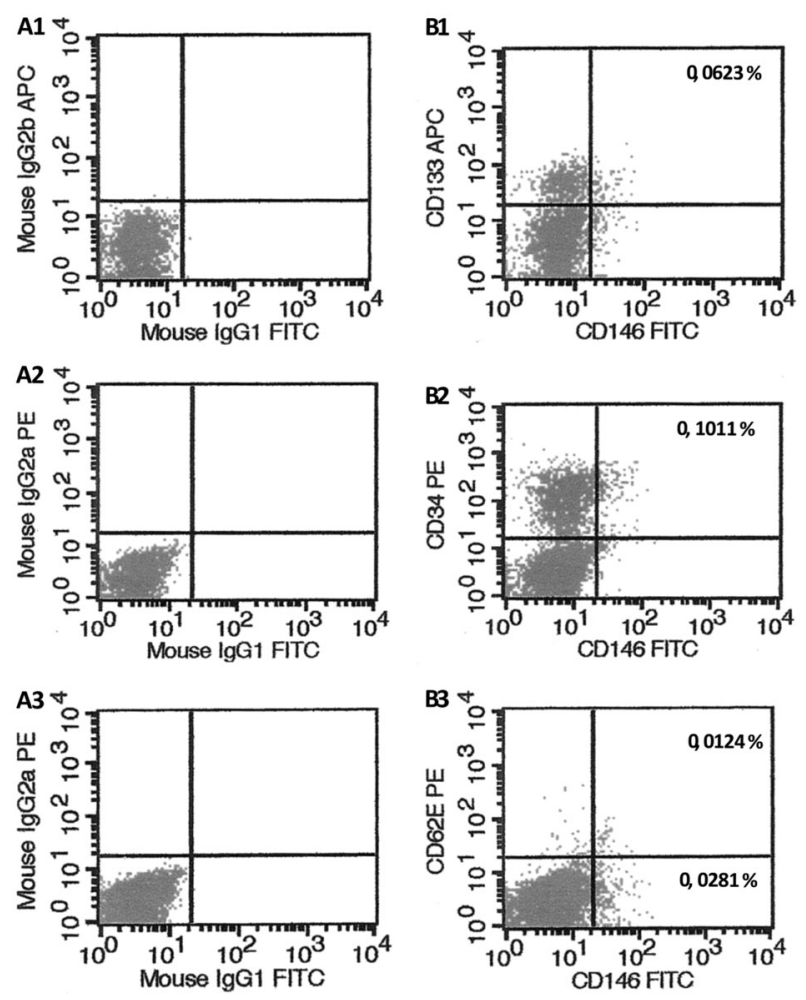

Figure 2. Flow cytometry analyses of endothelial cells in venous blood samples from chronic myeloid leukemia (CML) patients in blast crisis. $A 1, A 2$ and $A 3$, corresponding isotypic control. $B 1$, endothelial precursor cells (EPCs) expressing CD45-/dim/

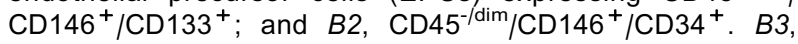
Characterization of activated mature circulating endothelial cells expressing CD45/dim/CD146 ${ }^{+} / \mathrm{CD}^{-} 2 \mathrm{e}^{+}$and $\mathrm{mCEC}\left(\mathrm{CD} 45^{-/ \mathrm{dim}} /\right.$ $\left.\mathrm{CD} 146^{+} / \mathrm{CD} \mathrm{e}^{-}\right)$. $4^{\circ} \mathrm{C}$ for $7400 \mathrm{~g}$, the supernatant was discarded, and the pelleted RNA was dried and resuspended in $50 \mu \mathrm{L}$ DEPCtreated distilled water. To detect RNA degradation, an aliquot of each total RNA sample was run on an $0.8 \%$ agarose gel stained with ethidium bromide (EtBr). RNA was quantified at $260 / 280 \mathrm{~nm}$ using a spectrophotometer (ND-1000; Nanodrop, USA). Treatment of RNA samples with DNAse was performed according to the kit manufacturer's instructions (M610A; Promega Corporation, USA).

Real-time quantitative PCR. Real-time quantitative PCR gene expression assay for VEGF was carried out using Taqman methodology (Hs00173626, Applied Biosystems, USA). We chose beta-glucuronidase (GUSB) as the control gene (4333767-F, Applied Biosystems). Amplifications were carried out in a volume of $12.5 \mu \mathrm{L}$ using $3 \mu \mathrm{L}$ of RNA (50 ng of RNA) and a SuperScript III Platinum One-Step Quantitative RT-PCR System kit (Invitrogen). The reactions were carried out in a Rotor-Gene RG 3000 thermal cycler (Corbett Research, Applied Biosystems) under standard conditions. Relative quantification was carried out using the comparative $\Delta \Delta \mathrm{CT}$ method following the procedure described by Livak and Schmittgen (17). All samples were analyzed in duplicate, and the median was used for further calculations. KG1, a myeloid cell line, was used as positive control, and calibrators were added to each plate.

\section{Statistical analysis}

Because of the non-normal distribution of the expression data (Shapiro-Wilk test), we used the nonparametric Kruskal-Wallis test and BioEstat 4.0 software (Sociedade Civil Mamirauá, Brazil) to compare medians and percentages of CEC subtypes and VEGF gene expression of the different phases of $C M L$ and healthy subjects. The significance level $(\alpha)$ was $5 \%$, and Wilcoxon's test was used to compare the results of duplicate assays.

\section{Results}

\section{CECs}

Fifty healthy subjects were analyzed, 25 (50\%) males and $25(50 \%)$ females with a median age of 46 years 
Table 1. Minimum and maximum percentages of CECs and VEGF gene expression.

\begin{tabular}{lrrrrr}
\hline & \multicolumn{2}{c}{ CEC (\%) } & & \multicolumn{2}{c}{ VEGF } \\
\cline { 2 - 3 } \cline { 5 - 6 } \cline { 5 - 6 } Min & Max & & Min & Max \\
\hline Healthy subjects & & & 0.030 & 0.760 \\
CEC & 0.0000 & 0.0530 & & \\
EPC & 0.0000 & 0.0265 & & \\
mCEC & 0.0000 & 0.0330 & & \\
aCEC & 0.0000 & 0.0133 & & \\
Blast crisis & & & 0.010 & 2.110 \\
CEC & 0.0011 & 18.9503 & & \\
EPC & 0.0000 & 3.7587 & & \\
mCEC & 0.0011 & 18.7066 & & \\
aCEC & 0.0000 & 0.0329 & & \\
Chronic phase & & & 0.060 & 1.150 \\
CEC & 0.0000 & 0.1557 & & \\
EPC & 0.0000 & 0.0344 & & \\
mCEC & 0.0000 & 0.1213 & & \\
aCEC & 0.0000 & 0.0251 & & \\
Accelerated phase & & & 0.020 & 3.220 \\
CEC & 0.0000 & 0.0604 & & \\
EPC & 0.0000 & 0.0586 & & \\
mCEC & 0.0000 & 0.0430 & & \\
aCEC & 0.0000 & 0.0116 & & \\
\hline
\end{tabular}

CEC: circulating endothelial cells; VEGF: vascular endothelial growth factor; EPC: endothelial precursor cells; mCEC: mature circulating endothelial cells; aCEC: activated circulating endothelial cells; min: minimum; max: maximum.

(range 19-79 years). The median percentages of CECs, EPCs, mCECs, and activated (a)CECs were $0.0059 \%$, $0.0023 \%, 0.0022 \%$, and $0.0021 \%$, respectively. The BC group consisted of 23 patients, 13 (59\%) males and 10 $(41 \%)$ females, with a median age of 52 years (range $20-74$ years). The median percentages of CECs, EPCs, mCECs, and aCECs were $0.0146 \%, 0.0035 \%, 0.0072 \%$, and $0.0022 \%$, respectively. There were $31 \mathrm{CP}$ cases, 18 (56\%) males and $13(42 \%)$ females, with a median age of 52 years (range 19-82 years). The median percentages of CECs, EPCs, mCECs, and aCECs were $0.0098 \%$, $0.0044 \%, 0.0033 \%$, and $0.0010 \%$, respectively. In the AP group, there were 30 patients, 16 (53.3\%) males and 14 $(46.7 \%)$ females, with a median age of 58 years (range 27-81 years). The median percentages of CECs, EPCs, mCECs, and aCECs were $0.0059 \%, 0.0023 \%, 0.0023 \%$, and $0.0011 \%$, respectively. The $\mathrm{BC}$ group had significantly higher percentages of CECs and mCECs than the control $(P<0.01)$ and AP $(P<0.01)$ groups. There were no significant differences in the percentages of CECs in the $\mathrm{CP}$ and AP groups and the healthy subjects. No significant differences were found in the results of duplicate CEC data acquired by flow cytometry (not shown). Minimum and maximum percentages of CECs are shown in Table 1.

\section{VEGF}

Efficiency of real-time PCR. The efficiency of real-time PCR was tested in serial dilutions of RNA from the KG1 cell line, demonstrating $98 \%$ efficiency for the VEGF gene with an $R^{2}$ of 0.99 and $100 \%$ efficiency for the endogenous GUSB gene with an $\mathrm{R}^{2}$ of 0.99 .

VEGF expression. Thirty-two healthy subjects were analyzed, $17(53.1 \%)$ males and 15 (46.9\%) females with a median age of 51 years (range 21-77 years). The median value of VEGF expression was 0.145 (range 0.030-0.760). The BC group consisted of 14 patients, 11 $(78.6 \%)$ males and $3(21.4 \%)$ females with a median age of 53 years (range 24-67 years). The median VEGF expression was 0.245 (range $0.010-2.110$ ). There were 25 cases in the CP group, $14(56 \%)$ males and $11(44 \%)$ females with a median age of 52 years (range 19-82 years). The median VEGF expression was 0.330 . There were 26 patients in the AP group, $12(46.1 \%)$ males and $14(53.9 \%)$ females with a median age of 58 years (range 26-81 years). The median VEGF expression was 0.320 . $V E G F$ gene expression was significantly higher in all phases of CML than it was in the healthy patients. There were no statistically significant differences in VEGF expression in the different phases of CML. Minimum and maximum percentages of VEGF gene expression are shown in Table 1.

\section{Discussion}

The initial study hypothesis was that a significant increase in CECs in peripheral blood occurred at the expense of EPCs in BC of CML. This increase was thought to be related to the increased vascular diameter observed at that stage and a result of neovascularization and increased microvascular density in the bone marrow microenvironment, which are directly related to mobilization of EPCs $(10,13)$. However, this hypothesis was not confirmed. The increase of CECs in peripheral blood during $\mathrm{BC}$ was at the expense of mCECs, leading us to believe that this CEC subtype may have been derived from the mobilization of newly formed vessels or by recruiting neighboring vessels to neoangiogenesis, with the release of mCECs. This activity has previously been demonstrated in murine tumor models (18).

To corroborate the CEC findings, we analyzed VEGF gene expression. This growth factor is an important mediator of angiogenesis and is involved in the pathogenesis of myeloid malignancies, including CML (19). However, there was an increase of VEGF in all phases of CML. Our results are consistent with previous studies demonstrating that the malignant cells of CML overexpress VEGF. Thus, this marker has not proven useful to discriminate the different stages of CML. However, we observed a significant increase in VEGF in myeloid BC compared to lymphoid $\mathrm{BC}$, corroborating the results reported by Krauth et al. (19). Those authors suggest 
that the lymphoid $\mathrm{BC}$ has a better prognosis than myeloid $B C$ in CML. Wierzbowska et al. (5) suggested that endothelial cells may enhance the survival and proliferation of leukemic blasts and mediate chemotherapy resistance in acute myeloid leukemia (AML). Those authors found a large numbers of CECs in the peripheral blood of AML patients, and their levels were correlated with disease status and response to therapy. Recently, Ibrahim et al. (8) demonstrated that aCEC and EPC levels were higher in AML patients than in healthy controls $(\mathrm{P}<0.0001$ and $\mathrm{P}<0.001$, respectively). They also reported that $C E C$ levels were correlated with disease status in AML.

In line with our results, Rigolin et al. (7) demonstrated increased numbers of EPCs in chronic lymphocytic leukemia (CLL) compared to healthy controls. Higher CEC levels were associated with more aggressive disease and shorter time to first treatment $(\mathrm{P}<0.001)$.

Our study found that quantification of CECs in peripheral blood was useful for predicting and identifying

\section{References}

1. Fidler IJ, Ellis LM. Neoplastic angiogenesis - not all blood vessels are created equal. $N$ Engl J Med 2004; 351: 215216, doi: 10.1056/NEJMp048080.

2. Khakoo AY, Finkel T. Endothelial progenitor cells. Annu Rev Med 2005; 56: 79-101, doi: 10.1146/annurev.med.56.0902 03.104149 .

3. Zammaretti $\mathrm{P}, \mathrm{Zisch} \mathrm{AH}$. Adult 'endothelial progenitor cells'. Renewing vasculature. Int J Biochem Cell Biol 2005; 37: 493-503, doi: 10.1016/j.biocel.2004.06.018.

4. Cortelezzi A, Fracchiolla NS, Mazzeo LM, Silvestris I, Pomati M, Somalvico F, et al. Endothelial precursors and mature endothelial cells are increased in the peripheral blood of myelodysplastic syndromes. Leuk Lymphoma 2005; 46: 1345-1351, doi: 10.1080/10428190500144235.

5. Wierzbowska A, Robak T, Krawczynska A, Wrzesien-Kus A, Pluta A, Cebula B, et al. Circulating endothelial cells in patients with acute myeloid leukemia. Eur J Haematol 2005; 75: 492-497, doi: 10.1111/j.1600-0609.2005. 00549.x.

6. Zhang H, Vakil V, Braunstein M, Smith EL, Maroney J, Chen $\mathrm{L}$, et al. Circulating endothelial progenitor cells in multiple myeloma: implications and significance. Blood 2005; 105: 3286-3294, doi: 10.1182/blood-2004-06-2101.

7. Rigolin GM, Maffei R, Rizzotto L, Ciccone M, Sofritti $O$, Daghia $G$, et al. Circulating endothelial cells in patients with chronic lymphocytic leukemia: clinical-prognostic and biologic significance. Cancer 2010; 116: 1926-1937, doi: 10.1002/cncr.24961.

8. Ibrahim KM, Ragab SH, Mohamed NAA, Emara NA, Alghafar NA, Kandeal E, et al. Circulating endothelial cells in acute myeloid leukemia. Aust J Basic Appl Sci 2011; 5: 1304-1311.

9. Sawyers CL. Chronic myeloid leukemia. N Engl J Med 1999; 340: 1330-1340, doi: 10.1056/NEJM199904293401706. early progression of $\mathrm{CML}$ to $\mathrm{BC}$, in contrast to VEGF, a variable that was high at all stages.

Despite the significant results observed in our study, we believe it is important to conduct a prospective study with patient follow-up from diagnosis through treatment to determine the percentages and monitor CEC types and VEGF gene expression. Thus, one could trace variations in those results and the impact of those variables at each stage in the progression of CML. In addition, such a study could assess, and increase our understanding of, the behavior of those variables in patients refractory to treatment and help to improve their management.

\section{Acknowledgments}

The authors would like to thank the Day Hospital of Hematology and Immunopathology Laboratory, Hospital das Clínicas, São Paulo. Research partially supported by FAPESP (06/55572-5) and Management Center of Hematology.

10. Musolino C, Alonci A, Bellomo G, Loteta B, Quartarone E Gangemi D, et al. Levels of soluble angiogenin in chronic myeloid malignancies: clinical implications. Eur $\mathrm{J}$ Haematol 2004; 72: 416-419, doi: 10.1111/j.1600-0609. 2004.00253.x.

11. Aguayo A, Kantarjian H, Manshouri T, Gidel C, Estey E, Thomas $\mathrm{D}$, et al. Angiogenesis in acute and chronic leukemias and myelodysplastic syndromes. Blood 2000; 96: 2240-2245.

12. Verstovsek $\mathrm{S}$, Kantarjian $\mathrm{H}$, Manshouri T, Cortes J, Giles FJ, Rogers A, et al. Prognostic significance of cellular vascular endothelial growth factor expression in chronic phase chronic myeloid leukemia. Blood 2002; 99: 22652267, doi: 10.1182/blood.V99.6.2265.

13. Korkolopoulou $P$, Viniou N, Kavantzas N, Patsouris E, Thymara I, Pavlopoulos PM, et al. Clinicopathologic correlations of bone marrow angiogenesis in chronic myeloid leukemia: a morphometric study. Leukemia 2003; 17: 89-97, doi: 10.1038/sj.leu.2402769.

14. Mancuso P, Burlini A, Pruneri G, Goldhirsch A, Martinelli G, Bertolini F. Resting and activated endothelial cells are increased in the peripheral blood of cancer patients. Blood 2001; 97: 3658-3661, doi: 10.1182/blood.V97.11.3658.

15. Mariucci S, Rovati B, Bencardino K, Manzoni M, Danova M. Flow cytometric detection of circulating endothelial cells and endothelial progenitor cells in healthy subjects. Int $J \mathrm{Lab}$ Hematol 2010; 32: e40-e48, doi: 10.1111/j.1751-553X.200 8.01105.x

16. Ozdogu H, Sozer O, Boga C, Kozanoglu L, Maytalman E, Guzey M. Flow cytometric evaluation of circulating endothelial cells: a new protocol for identifying endothelial cells at several stages of differentiation. Am J Hematol 2007; 82: 706-711, doi: 10.1002/ajh.20904.

17. Livak KJ, Schmittgen TD. Analysis of relative gene expression data using real-time quantitative PCR and the 
2(-Delta Delta C(T)) method. Methods 2001; 25: 402-408, doi: 10.1006/meth.2001.1262.

18. Rafii S, Lyden D, Benezra R, Hattori K, Heissig B. Vascular and haematopoietic stem cells: novel targets for anti-angiogenesis therapy? Nat Rev Cancer 2002; 2: 826-835, doi: 10.1038/ nrc925.
19. Krauth MT, Simonitsch I, Aichberger KJ, Mayerhofer M Sperr WR, Sillaber C, et al. Immunohistochemical detection of VEGF in the bone marrow of patients with chronic myeloid leukemia and correlation with the phase of disease. Am J Clin Pathol 2004; 121: 473-481, doi: 10.1309/ 3JLTFNNEDQHB4AOP. 